ArtefaCToS. Revista de estudios de la ciencia y la tecnología

eISSN: $1989-3612$

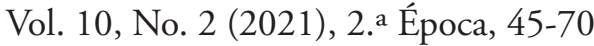

DOI: https://doi.org/10.14201/art20211024570

\title{
La perspectiva esencialista en la concepción de la tecnología
}

\section{The Essentialist Perspective in the Conception of Technology}

\author{
Nicolás VILOUTA RANDO*; Pablo A. PELLEGRINI** \\ * Instituto de Estudios sobre la Ciencia y la Tecnología, \\ Universidad Nacional de Quilmes, Argentina \\ viloutar@gmail.com
}

${ }^{* *}$ CONICET / Instituto de Estudios sobre la Ciencia y la Tecnología, Universidad Nacional de Quilmes-CIC-BA, Argentina

ppellegrini@unq.edu.ar

Recibido: 18/04/2021. Revisado: 25/07/2021. Aceptado: 17/08/2021

\section{Resumen}

El pesimismo tecnológico es una perspectiva que, desde mediados del siglo XX, se popularizó tanto en círculos intelectuales como en la cultura popular, convirtiéndose en una de las maneras más comunes de pensar la tecnología. En el presente trabajo caracterizaremos el estilo de pensamiento esencialista, mostrando de qué manera permea dicha perspectiva de la tecnología, dotando a esta de autonomía y considerándola una fuente intrínseca de consecuencias nefastas, tanto para la sociedad como para la naturaleza. Se hará una reconstrucción histórica de la perspectiva esencialista, caracterizando su formulación en el pensamiento filosófico de Aristóteles. A partir de allí, se indagará en el modo en que la perspectiva esencialista se despliega frente a la tecnología tanto en círculos académicos como en la cultura popular. Sobre el final, se expondrá el caso de los organismos genéticamente modificados (OGM) como un espacio de debate donde podremos ver el estilo de pensamiento esencialista movilizado.

Palabras clave: esencialismo; esencialismo tecnológico; pesimismo tecnológico; autonomía tecnológica; determinismo tecnológico; OGM; estilo de pensamiento. 


\begin{abstract}
Technological pessimism is a perspective that, since the middle of the 20th century, became popular both in intellectual circles and in popular culture, becoming one of the most common ways of thinking about technology. In this paper we will characterize the essentialist thought style, showing how it permeates this perspective of technology, endowing it with autonomy, and as an intrinsic source of dire consequences, both for society and for nature. A historical reconstruction of the essentialist perspective will be made, characterizing its formulation in Aristotle's philosophical thought. From there, we will investigate the way in which the essentialist perspective is deployed before technology in academia, as well as in popular culture. Finally, the case of genetically modified organisms (GMOs) will be presented as a space of debate where we can see the essentialist thought style mobilized.
\end{abstract}

Keywords: essentialism; technological essentialism; technological pessimism; technological autonomy; technological determinism; GMOs; styles of thought.

\title{
1. Introducción
}

En las formas de representación sobre las nuevas tecnologías y su relación con la sociedad, es frecuente la convicción de que esos desarrollos tecnológicos tendrán efectos inevitables sobre la salud o el ambiente más allá de cualquier contexto o intervención sobre la tecnología. Esta convicción puede encontrarse tanto en ámbitos académicos como en movimientos sociales y en la arena pública. Este estilo de pensamiento puede presentarse así en diversas controversias y debates sobre los impactos de los desarrollos científico-tecnológicos. Un estilo de pensamiento implica una comunidad de personas que comparten ciertas asunciones, aun cuando no sean conscientes de ello y, por ende, esas asunciones raramente son cuestionadas. Un estilo de pensamiento distintivo, según Mary Douglas $(1998,14)$, "se desarrolla como el género comunicativo que le permite a una unidad social hablarse a sí misma de sí misma y así constituirse”. La existencia de estilos de pensamiento nos permite dar cuenta de que hay distintas maneras de pensar un mismo fenómeno, y que cada forma particular de pensarlo está condicionada por elementos culturales (Pellegrini, 2019). Llamaremos esencialismo a un estilo de pensamiento cuya explicación sobre las cosas se asienta en la existencia de una naturaleza intrínseca de la cosa que la define, que marca su identidad y determina su comportamiento más allá de los accidentes que pueda encontrar en su desarrollo. Se trata de una concepción frecuente de la tecnología, toda vez que:

para el esencialismo, las singularidades de cada tecnología se deben, a lo sumo, a meras contingencias, pero es su naturaleza primaria, su esencia, lo que define lo que "es" la tecnología en sus rasgos principales. Donde hay 
una "esencia" es porque se atribuye una naturaleza intrínseca que explica al objeto más allá de lo que le infrinja la realidad externa. (Pellegrini, 2013, 36)

En este trabajo procuraremos brindar algunos elementos que permitan caracterizar al esencialismo. Para ello, comenzaremos haciendo una reconstrucción histórica de la perspectiva esencialista, caracterizando su formulación en el pensamiento filosófico de Aristóteles. A partir de allí, indagaremos en el modo en que la perspectiva esencialista se despliega frente a la tecnología. Sobre el final, expondremos el caso de los organismos genéticamente modificados (OGM) como un espacio de debate donde podremos ver la perspectiva esencialista movilizada.

\section{Los orígenes filosóficos del esencialismo}

Más allá de la diversidad de matices y controversias que implican cada una de sus versiones, podemos definir al esencialismo — siguiendo a Oderberg (2007) _ como una postura que considera que las cosas tienen una esencia - la cual determina su definición y clasificación- que no pertenece al lenguaje o a las convenciones, sino que hace a la constitución misma de la realidad. Esta es una doctrina filosófica que se remonta a la antigüedad y posee numerosas encarnaciones que han surgido a lo largo del tiempo. Sin embargo, podemos identificar en Aristóteles - sino la primera- la tradición más difundida e influyente del esencialismo, que al día de hoy sigue siendo discutida e interpretada siempre que se aborda esta doctrina.

Aristóteles desarrolla su propuesta esencialista en el trabajo esotérico conocido como Metafísica, donde aborda la pregunta por el ser. Pero a diferencia de lo realizado desde otras disciplinas, aquí las indagaciones girarán en torno al ser en cuanto ser o - dicho de otra manera - "a lo que es, en tanto que algo que es" (1003a 21). Así, por ejemplo, el hombre puede ser estudiado desde la física en cuanto móvil o sujeto de cambio, o bien desde la política en cuanto animal social, pero en todos los casos estaremos hablando de un mismo ser, descrito de distintas maneras. Todas estas descripciones son predicados de un mismo ente (en este caso el hombre), lo que lleva a Aristóteles a decir que "el ser se dice de muchas maneras" (1030a 19). Además, las personas pueden cambiar, a través de la pérdida y ganancia de propiedades, pero sin que por eso dejen de ser personas. Será la tarea de la metafísica, también llamada filosofía primera, estudiar a todo ser - no solo al hombre- en cuanto sujeto último de predicación, más allá de cualquier otra propiedad o cambio accidental (1003a 30).

Entre las cosas que existen en el universo, Aristóteles distinguirá y priorizará ontológicamente a las sustancias, ya que son el sujeto último de predicación —es decir, aquello de lo que puede ser predicado pero no es predicado de nada-, entre los que reconoce a los animales y sus partes, las plantas y los cuerpos simples (Ross, 1981). Las otras categorías que conforman el universo aristotélico tendrán 
una existencia secundaria, ya que solo pueden existir si son predicadas de alguna sustancia particular, como el color, la cantidad o la relación (Cohen, 2020). Al preguntarse por la identidad de las sustancias, Aristóteles buscará distinguir entre las propiedades accidentales de estas y aquellas que son esenciales, las que las hacen ser una determinada sustancia y no otra. De esta manera, la pregunta por el ser se convierte en la pregunta por la sustancia y, en última instancia, en la pregunta por la esencia: ¿qué es aquello que define a una sustancia más allá de sus características accidentales? (Cohen, 2020).

Esta indagación también buscará dar respuesta al interrogante por el cambio, tanto el accidental como el sustancial. Intentará dar sentido al hecho de que la realidad está continuamente mutando, con cosas que comienzan a existir mientras otras cesan de hacerlo; o bien cosas que pierden algunas propiedades y ganan otras. En todo cambio, señala Aristóteles, debe haber tres cosas: 1. algo que persiste a través de todo el proceso, 2. algo que se pierde y 3. algo que se adquiere, funcionando estas dos últimas como pares opuestos (Ainsworth, 2020). En el caso de los cambios accidentales, aquello que permanece constante es la sustancia, lo que da identidad a lo que sufre el cambio; mientras que los pares opuestos que se pierden/obtienen son propiedades, que pertenecerán a alguna de las categorías secundarias que señala Aristóteles (como cantidad, cualidad, posición, etc.). En un cambio sustancial, la sustancia no se conserva, sino que se transforma en otra. Por ejemplo, si pintamos una pared de rojo, esta habrá ganado una propiedad, pero seguirá siendo una pared. En cambio, si tiráramos la pared abajo, los ladrillos que la conforman - la materia - no desaparecen, pero aquello que queda ya no es más una pared, sino una pila de escombros (Oderberg, 2007). El hecho de que la sustancia haya cambiado su identidad, pero la materia permanezca, lleva a preguntarnos qué es lo que cambió en esta transformación. ¿Qué es lo que hacía a la pared una pared y que después de derribarla ya no está? ¿Cuál es la esencia de la pared? No puede ser - al menos no únicamente- la materia de la que está compuesta, ya que esta permaneció constante. Aristóteles señalará un segundo componente que dará identidad al ente - determinando qué sustancia es- y constituirá una unidad con la materia: la forma.

La tesis de que toda sustancia es un compuesto de materia y forma es bautizada por Aristóteles como hilemorfismo. Una analogía para ilustrar esto es entender una estatua de mármol como compuesta por mármol (sustancia) y un ordenamiento determinado de este (forma) (Cohen, 2020). Es importante no confundir el concepto de forma con el de la simple silueta, cosa que la analogía con la estatua puede inducir. Aristóteles utiliza un término para referirse a la configuración sensible de un objeto - morphê- y otro distinto para hablar de lo que considera la estructura inteligible donde reside la naturaleza íntima de una cosa, que señala como eidos (Ross, 1981). Esta segunda acepción será la que el hilemorfismo tome como la esencia de una sustancia. 
De esta manera, la teoría hilemórfica permite entender tanto los cambios accidentales como los sustanciales, pudiendo señalar en ambos qué permanece, qué se pierde y qué se obtiene. En los cambios accidentales - como una estatua que se pinta- la sustancia permanece a lo largo de todo el cambio, ya que la materia y forma se conservan de manera inalterada. Lo que se pierde y lo que se adquiere en estos casos son propiedades que pertenecen a las categorías secundarias, como cantidad o cualidad. En los cambios sustanciales - como un bloque de mármol que se cincela para hacer una estatua-, dado que la sustancia es el producto del cambio, esta no puede ser la que persista durante todo el proceso. El hilemorfismo señala a la materia como el sustrato que persiste en el cambio sustancial y a la forma como aquello que se pierde para ser reemplazada por otra distinta, lo que dará lugar a una nueva sustancia.

En síntesis, el hilemorfismo propone que toda sustancia es una unidad de materia y forma, donde la primera permanece a lo largo de todo cambio, mientras que la segunda puede ser identificada con la esencia de aquella. En otros términos -que Aristóteles utiliza en su Física - la materia es pura potencia, mientras que la forma es acto. La forma actualiza y limita la potencialidad, diferenciando una realidad indiferenciada. Recíprocamente, la materia limita a la forma, restringiéndola a determinadas regiones de la realidad (Oderberg, 2007), permitiendo diferenciar individuos que pertenecen a un mismo tipo de sustancia (Ross, 1981).

Sin embargo, a pesar de considerar a las sustancias compuestos hilemórficos y ser productos de una interacción simultánea entre materia y forma, a esta última le es otorgada cierta prioridad sobre la materia, ya que la forma es el fin (telos) por el cual la materia - la potencialidad - existe y hacia donde se dirige $^{1}$ (Gómez-Lobo, 1996; Cohen, 2020). Aristóteles rechaza así la idea de que las cosas se hayan formado desde una lógica aditiva o progresiva, desde lo más simple a lo más complejo, ya que la finalidad de una sustancia estaría inscrita en su propia esencia (Gómez-Lobo, 1996). Y es que la forma posee un carácter holístico que impide concebirla de manera reduccionista o materialista, definiéndola simplemente como la sumatoria de sus componentes materiales o la relación interna de estos. El esencialismo advierte que no podemos conocer o aprehender la esencia de una cosa limitándonos a la enumeración de sus partes y propiedades -incluso cuando estas sean esenciales-, sino que debemos alcanzar la forma de esta, la cual no se encuentra en la mera suma de propiedades. El enfoque holista -al advertir que el todo determina las características de las partes- permite al esencialismo plantear la relación inversa: será la forma de una sustancia la que determine y limite las características y comportamiento de sus componentes, así como sus propiedades esenciales, y no al revés (Oderberg, 2007). Retomando el ejemplo de

\footnotetext{
${ }^{1}$ Las explicaciones teleológicas son fundamentales en la filosofía aristotélica, y es una de las cuatro causas que describe junto con las causas materiales (materia), formales (forma) y eficientes. Si bien esto pareciera solaparse y contradecir la teoría hilemórfica, en el caso de las sustancias, podemos ubicar sus causas eficientes y finales en la forma.
} 
la estatua, esta comenzará a existir en el exacto instante en que el escultor aplique sobre el mármol la última cincelada, ya que "lo que deviene deviene por partes, pero la forma no tiene partes: es la estructura del conjunto" (Ross, 1981, 210). Como explica Ross, la forma "no es otro elemento de la cosa sobreańadido a sus elementos materiales, ni un compuesto de estos elementos; es lo que hace que ciertos elementos sean carne y otros una sílaba” (Ross, 1981, 206)².

Oderberg (2007) da un ejemplo esclarecedor para entender el carácter holístico de la forma, al plantear la imposibilidad de concebir a un perro como la suma de un cuerpo (la materia) más el añadido de la forma o esencia canina. Si esta lógica aditiva fuera correcta, sería sencillo imaginar que - en caso que nuestro perro muera- podríamos traer a nuestra mascota de vuelta a la vida con solo volver a agregar la forma canina a su cadáver. Sin embargo, un cadáver no es un cuerpo vivo al que le falta algo, no es materia en busca de una forma, sino una sustancia completamente distinta, con forma propia. Dicha forma será la causa de una serie de nuevas funciones de decaimiento y desintegración que comenzarán a ocurrir en el mismo instante de la muerte. En otras palabras, la forma no se agrega a la materia, sino que permea enteramente a la sustancia que la posee, tanto horizontalmente, en cada una de sus partes, como verticalmente, hasta las partículas elementales que la constituyen (Oderberg, 2007).

Este modo de entender la naturaleza de toda sustancia, cuya forma será el origen de toda propiedad esencial, así como la que dirigirá su comportamiento, será el fundamento metafísico para entender y sostener la cosmovisión aristotélica (Ellis, 2002). Según esta concepción, mantenida en buena parte del mundo occidental hasta el siglo XVI, el universo está organizado en esferas celestes concéntricas y compuesto en última instancia por cuatro elementos - tierra, aire, fuego y agua - que constituyen todo lo que existe debajo de la esfera lunar, mientras que un quinto elemento - el éter- es la sustancia que compone todo lo que existe más allá de dicha esfera. Las esencias de dichos elementos son las que permiten explicar su comportamiento y organización. Por ejemplo, la caída y trayectoria natural de los objetos pesados - como una roca - cobran sentido al señalar la tendencia de su principal componente -el elemento tierra- a ubicarse en el centro del universo. Esta esencia del elemento tierra será también la que explique la ubicación de nuestro planeta en el centro del universo. De igual manera, también subyace el enfoque esencialista en la clasificación y jerarquía de los seres vivos, en cuyo último escalón se encontraría el hombre, gracias a su esencia -que residiría, como en todo ser vivo, en el alma- que le hace ser racional y buscar la virtud y sabiduría (Ellis, 2002).

\footnotetext{
${ }^{2}$ Ross aclara que para Aristóteles la esencia no es un componente material que existiría al lado de otros componentes materiales, sino el principio estructural de la cosa concreta (Ross, 1981, 206-207).
} 


\section{La perspectiva esencialista en la reflexión sobre la tecnología}

A partir de esta revisión de la tradición filosófica del esencialismo, nos interesa ahora mostrar de qué manera algunas de sus ideas se encuentran presentes a la hora de abordar y pensar la tecnología. En particular, nos interesa explorar los modos de entender la tecnología que se sostienen en postulados esencialistas, pero sin necesariamente adscribir al esencialismo como doctrina metafísica. Según esta postura, que llamaremos esencialismo tecnológico, la tecnología poseería una esencia inmutable que dicta su desarrollo y características, de manera completamente independiente de todo factor exterior.

La perspectiva esencialista, que explica los fenómenos en base a cualidades absolutas, está en las antípodas de las explicaciones relativistas, las cuales analizan los fenómenos en relación a su contexto. En ese sentido, los estudios sobre ciencia, tecnología y sociedad han sido receptivos a perspectivas más relativistas. En particular, la sociología del conocimiento científico (SSK) se desarrolló en oposición al racionalismo, esencialismo y fundacionalismo filosófico (Shapin, 1995, 297). Así, autores como Barnes, Bloor o Collins señalaron que el conocimiento no debe explicarse a través de categorías como lo racional o verdadero, pues no hay conocimientos o verdades absolutas (Barnes y Bloor, 1982; Collins, 1981; Bloor, 2011). Aunque renegando del relativismo, también Latour opone su particular historicismo al esencialismo y a la idea de que los objetos son invariantes (Latour, 2000; Kuukkanen, 2016). De todos modos, el esencialismo tecnológico no suele ser objeto de revisión, y en particular aquí proponemos indagar en los rasgos principales del esencialismo como estilo de pensamiento que condiciona la mirada sobre la tecnología.

En particular, nos interesará abordar la vertiente pesimista de dicha perspectiva, especialmente influyente desde la segunda mitad del siglo XX. Según esta tradición, la sociedad estaría siendo conducida por la tecnología por un camino plagado de peligros y a un destino trágico, de alienación, esclavitud y destrucción. La tecnología es considerada "una amenaza independiente y esencialmente peligrosa a la existencia humana y al planeta" (Scott, 2018, ix). En este apartado analizaremos de qué modos concretos se manifiesta este tipo de representación y cómo el esencialismo subyace en ella.

Desde mediados del siglo XX, se ha popularizado y arraigado en el sentido común de buena parte de la sociedad la idea de que la tecnología - especialmente la tecnología moderna- posee inherentemente una serie de características que la llevan a dominar cada vez más a la sociedad, degradándola moral y espiritualmente y conduciéndola a su propia destrucción. La explicación a este fenómeno - postula esta mirada - debe buscarse en la naturaleza propia de la tecnología, en su esencia, que vuelve inevitables todas sus consecuencias negativas. 
En ese sentido, aquí proponemos caracterizar el esencialismo tecnológico no como una tradición filosófica metafísica, sino como algo más amplio, esto es, como un estilo de pensamiento que, en tanto tal, se manifiesta en distintos espacios de la cultura, desde producciones académicas hasta expresiones populares.

\subsection{Reacción al optimismo tecnológico}

Esta perspectiva se enfrenta y nace como reacción al optimismo tecnológico, que fue probablemente el lugar más común desde el cual se juzgó a la tecnología hasta la mitad del siglo pasado y posee aún una amplia aceptación en ciertos sectores de la sociedad. Apoyado en la idea de progreso del iluminismo y el positivismo decimonónico, la tecnología era comprendida como el fruto de la racionalidad humana puesta al servicio de objetivos y problemas específicos. En principio no se la juzgaba como mala ni buena, sino puramente instrumental y neutral desde el punto de vista social y político (Achterhuis, 2001). Al igual que la ciencia - y como resultado directo de la aplicación de esta- la tecnología era considerada una fuente de progreso social y mejoras constantes.

La visión optimista de la tecnología se vio reflejada en las primeras reflexiones sistemáticas sobre la tecnología, en lo que Mitcham (1989) llama la filosofía de la tecnología ingenieril. Esta corriente, que reflexionaba sobre la tecnología "desde dentro", no solo analiza la naturaleza de la tecnología, sino que busca explicar el mundo en términos tecnológicos (Mitcham, 1989). Sostiene que humanidad y tecnología están signadas por una relación armoniosa, donde esta es fundamental en la completa realización de aquella. Por ejemplo, Ernst Kapp —fundador del término filosofía de la tecnología- consideraba que la tecnología era una proyección de los órganos y el alma humana sobre el mundo, humanizando a este y aliviando la existencia de las personas en él. La tecnología no posee una esencia más allá del ser humano, sino que es aquello propio del ser humano que se proyecta en la herramienta: la muela en el molino, el cristalino en un telescopio, el puño en un martillo, etc. (Kapp, 1998). De esta manera, "el hombre, en la herramienta, siempre se muestra a sí mismo" (Kapp, 1998, 2). Friedrich Dessauer, otro filósofo de esta corriente, considera el acto de creación técnica como la mejor vía para alcanzar la trascendencia y a la "cosa en sî" que es la realidad. La tecnología no solo traería beneficios materiales, sino un crecimiento ético, moral y místico, una experiencia religiosa que permite al hombre participar de la creación (Mitcham, 1989). La cultura popular también se vio impregnada de este optimismo, manifiesto - por ejemplo- en la vertiente utópica de la ciencia ficción y en las diversas exposiciones mundiales hechas desde mediados del siglo XIX, destinadas a mostrar los avances realizados en tecnología e innovación alrededor del mundo.

Sin embargo, durante la segunda mitad del siglo XX el optimismo tecnológico comienza a ser duramente cuestionado. Los usos nefastos dados a la tecnología para el exterminio de personas durante el régimen nazi, las bombas atómicas 
arrojadas sobre el pueblo japonés, los efectos indeseados de la energía nuclear y de pesticidas como el DDT, fueron algunos de los problemas cuya amplia repercusión comenzaron a evidenciar que la tecnología moderna no podía igualarse automáticamente al progreso social. Así, por ejemplo, desde los movimientos ambientalistas que nacen en la década del 70', comienza a considerarse a la tecnología moderna como un elemento de riesgo y sospecha en sí mismo, que se opone y amenaza a la naturaleza y al medioambiente (Pellegrini, 2013, 29-33). En el ámbito de la literatura, las utopías dejan lugar a las distopías o utopías negativas, las cuales "parecen enfatizar el elemento deshumanizante, hasta monstruoso, de la civilización industrial" (Capanna, 1966, 190). Diversas obras de ciencia ficción manifiestan así que la tecnología moderna poseería efectos devastadores en la naturaleza y el ser humano: ya sea la televisión y la consecuente destrucción de la literatura, en Fahrenheit 451 de Ray Bradbury; los desechos industriales que provocan una escasez de agua global y ponen al mundo al borde de la destrucción, en La sequía de J. G. Ballard; o la biotecnología y el descontrol ecológico de animales modificados genéticamente, en Oryx y Crake de Margaret Atwood. Desde el ámbito académico, podemos encontrar las distopías intelectuales (Pellegrini, 2013, 33-38), descritas por la vertiente humanista de la filosofía de la tecnología, en autores como Martin Heidegger, Jacques Ellul o Lewis Mumford, a los que se suele englobar como pesimistas tecnológicos (Gómez, 1997; Parente, 2006; Gómez Di Vincenzo, 2012). En todos estos casos, es posible encontrar una caracterización de la tecnología que hace de esta una entidad amenazadora y alienante, cuyo avance no puede ser controlado ni sus consecuencias previstas.

Quintanilla considera que la mirada pesimista de la tecnología se debe al diseño opaco que muchas tecnologías suelen presentar. El usuario se siente tan ajeno o incapaz de comprender los procesos que encierran las tecnologías, que le generan -argumenta Quintanilla $(2017,19)$ - un extrañamiento, una alienación que lo lleva a asumir que la tecnología es incontrolable. Como el diseño de la tecnología estaría en la raíz de la respuesta pesimista, Quintanilla propone diseñar tecnologías más cercanas al usuario, tecnologías sobre las que sienta que puede ejercer el control y hacerse responsable, tecnologías que denomina "entrañables" (Quintanilla, 2017, 27). Sin negar el papel que puede desempeñar el diseño de una tecnología en la percepción que genera en el usuario, nuestro análisis se centra en el rol de la cultura como matriz que otorga un determinado sentido a los fenómenos que enfrenta, en este caso, a la tecnología. De tal forma, los estilos de pensamiento son patrones culturales arraigados en una sociedad y que funcionan como un prisma a través del que se interpreta la realidad. Analizar el esencialismo como un estilo de pensamiento nos permite dar cuenta de los condicionamientos culturales que pueden operar como marco de sentido para comprender una tecnología.

Asumir entonces que una tecnología tiene cualidades nocivas y que esas cualidades no serían producto de las circunstancias, propias de un contexto histórico específico, sino que estarían en la naturaleza intrínseca de esa tecnología, es un 
rasgo distintivo del pensamiento esencialista. Se trata así de una perspectiva que "interpreta los fenómenos históricos específicos en términos de una construcción conceptual transhistórica" (Feenberg, 2013, 24). De esta manera, la tecnología moderna poseería una esencia - un núcleo inalienable-que, como en la doctrina tradicional esencialista, explica su comportamiento y consecuencias sin importar el tiempo o espacio donde se despliegue.

En el siguiente apartado, caracterizaremos los rasgos de esta esencia atribuida a la tecnología moderna, con presencia en el ámbito académico y en la cultura popular.

\subsection{Autonomía y determinismo en la perspectiva esencialista}

Si bien la concepción de la tecnología recién descrita se ha popularizado en las últimas décadas, sus orígenes pueden rastrearse a más de 200 ańos, con el surgimiento del romanticismo, un movimiento cultural que nació como reacción a la industrialización y sus consecuencias. Uno de sus exponentes, el filósofo Jacques Rousseau, criticó el desarrollo y la civilización iluminista de su tiempo - principalmente encarnadas por la ciencia y la tecnología- por considerarlas perjudiciales para la moral y la sociedad. En contraste, halagó a las civilizaciones antiguas como superiores en espíritu, frente a la miseria, contaminación y degradación moral que caracterizaban a las ciudades industrializadas, situaciones denunciadas también en novelas de autores como Dickens y De Quincey. El romanticismo consideraba que el enfoque cuantitativo y analítico de las ciencias, impulsado en la idea de racionalidad del iluminismo, empobrecía la percepción de la realidad, relegando las emociones y la riqueza y exuberancia de los sentidos. Desde esta mirada, se consideraba que la tecnología estaba destruyendo la naturaleza y el espíritu humano (Dusek, 2006). Como expresa Mitcham (1989), la tecnología moderna es entendida desde la crítica romántica como oscurecedora y encubridora de los elementos esenciales de la vida. En cierta manera, pareciera ser la fuente del Mal. El poeta William Blake, por ejemplo, hablaba de los "molinos oscuros y satánicos" en su poema Jerusalem. A la tecnología y la corrupción que esta generaba, fue enfrentada la naturaleza, que comenzó a ser valorada como símbolo de pureza, sabiduría e inspiración (Dusek, 2006). Otra autora de la época, Mary Shelley, simboliza en su novela Frankenstein o el Moderno Prometeo no solo el carácter destructivo y abominable de la tecnología, sino cómo esta tiene vida propia, revelándose a la voluntad de su creador y provocando su perdición. Sin embargo, no toda mirada pesimista sobre la tecnología encierra necesariamente una concepción esencialista de la misma. Algunas críticas están dirigidas más a una racionalidad instrumental que a la tecnología en sí misma.

Es en las críticas del sacerdote y escritor inglés Samuel Butler donde encontramos uno de los primeros y más nítidos rechazos a la tecnología en base a una concepción eminentemente esencialista. Inspirado por El origen de las especies de 
Charles Darwin, en 1863 Butler publica el artículo Darwin among the machines [Darwin entre las máquinas]. En este ensayo, se considera a las máquinas como sujetas a un proceso evolutivo similar al descrito por Darwin en los seres vivos. Así como las plantas y animales surgieron mediante un lento proceso a partir de materia mineral e inerte, estaríamos frente a los albores de la "vida mecánica" y el surgimiento de un "reino mecánico", las máquinas podrán auto-perpetuarse y alcanzarán el rol de supremacía que hoy ostenta la especie humana:

estamos diariamente otorgándoles más poder y suministrándoles a través de artificios ingeniosos ese poder de autorregulación y autonomía que será para ellos lo que el intelecto ha sido para la raza humana. En el transcurso de los siglos nos visualizaremos como la raza inferior. (Butler, 1914, 182)

Butler termina su manifiesto llamando a que los humanos declaren la guerra a las máquinas, destruyendo todas y cada una de ellas antes que nos dominen. Estas ideas serán posteriormente ampliadas en su novela utópica Erewhon, donde dedica tres capítulos a desarrollar y describir el ficticio "Libro de las máquinas", que habría inspirado a la sociedad que describe Butler a destruir todas sus máquinas (Butler, 2012). Es posible identificar aquí un lugar común del esencialismo tecnológico y de sus críticas hacia la tecnología, cuya influencia puede apreciarse en muchos autores del siglo XX: la autonomía tecnológica.

La tesis de la autonomía tecnológica sostiene que la tecnología es independiente de todo control o decisión humana y que su desarrollo se da en función de una lógica propia (Dusek, 2006). Como señala Diéguez Lucena (2005), esto le da a la tesis un estatuto ontológico, toda vez que decreta que la tecnología es, por su propia naturaleza, ingobernable. Esta perspectiva se tornó muy popular en el siglo XX, encontrando en el filósofo de la tecnología Jacques Ellul uno de sus principales defensores. Pero yendo más allá de la ingobernabilidad de la tecnología, Ellul señala que esta "depende últimamente de sí misma, traza su propia ruta, es una fuerza primaria y no secundaria, debe ser considerada un "organismo" tendiente al cierre y la auto-determinación: es un fin en sí misma. La autonomía es la propia condición del desarrollo tecnológico" (Ellul, 2014, 430). Así, la tecnología deja de ser considerada un medio para alcanzar determinados fines externos a esta, sino que depende de una lógica propia, ajena al hombre. $\mathrm{Al}$ igual que con el comportamiento de las sustancias en la doctrina esencialista, las leyes y el telos que guían el desarrollo tecnológico deberían buscarse en la misma tecnología y no en el contexto en el cual se desenvuelve. Ellul sostiene que el estado, el poder político o el poder económico pueden frenar o retrasar el desarrollo tecnológico, pero esto no impide que la tecnología se siga comportando en base a leyes e imperativos propios. En el mejor de los casos, aquellos serán meros obstáculos temporales, intentos de detener el avance tecnológico que, a la larga, están irremediablemente condenados (Ellul, 2014). Ellul plantea una mirada fatalista, donde el imperativo tecnológico es imposible de frenar. 
Así como Marx estudió la economía y estructura capitalista para entender la sociedad de su tiempo, Ellul considera que para hacer lo mismo en el siglo XX es necesario analizar la técnica (Ellul, 1981; Mitcham, 1989). Y es que la misma, con su criterio de eficiencia, permea e invade todas las demás esferas de la actividad humana, en lo que Ellul llama el fenómeno técnico moderno (Gómez Di Vincenzo, 2012). Dicho fenómeno, que surge con la revolución industrial, ha llevado a la mecanización de la vida humana (Gómez Di Vincenzo, 2012) y convirtió a las personas en meras piezas de una maquinaria mucho más amplia. A partir de la complejización del sistema tecnológico, nadie tiene el conocimiento suficiente para comprenderlo enteramente: ni los ingenieros, a quienes se les escapan todas las consecuencias que vayan más allá de la esfera técnica (consecuencias políticas, sociales, económicas, etc.); ni los políticos, que son totalmente ignorantes del funcionamiento de las diversas tecnologías; ni mucho menos el público lego, desinformado en ambos aspectos (Dusek, 2006). Es en parte por esto que la tecnología siempre trae aparejados problemas imprevistos, al mismo tiempo que obliga a la sociedad a adaptarse a estos, en lugar de adaptar la tecnología. Esto último es lo que Langdom Winner (1977) llama adaptación reversa, lo que se ve facilitado a partir de los componentes sociales del sistema tecnológico, como la propaganda y el marketing.

Theodore Kaczyinski (más conocido como Unabomber), matemático y filósofo tristemente célebre por llevar a cabo una serie de ataques terroristas mediante cartas bomba a distintos profesores universitarios de Estados Unidos y a un avión, también encuentra en el sistema tecnológico desarrollado a partir de la revolución industrial la principal problemática de la sociedad actual. Notoriamente influenciado por Ellul (Kintz, 2014), Kaczynski desarrolla buena parte de la perspectiva descrita anteriormente en su famoso manifiesto La sociedad industrial y su futuro, declarando que la humanidad es sometida a las necesidades del sistema tecnológico, el cual evoluciona independientemente de cualquier factor ideológico (Kaczynski, 2008).

La metáfora del hombre como un engranaje, que utilizan Mumford (2014), Kaczynski (2008), o el mismo Ellul, son imágenes comunes en estas conceptualizaciones. Y es que la autonomía tecnológica suele estar estrechamente ligada a una segunda tesis: el determinismo tecnológico ${ }^{3}$. El mismo consiste en considerar que la relación entre tecnología y sociedad es asimétrica y unidireccional, con la primera como agente estructurante de la segunda (Dusek, 2006). De esta manera, la tecnología no solo se considera autónoma, sino que dicha autonomía termina sofocando y eliminando la libertad humana. El desarrollo tecnológico

\footnotetext{
${ }^{3}$ Las relaciones entre autonomía y determinismo tecnológico son el foco de discusión de numerosos trabajos y existen distintas formas de relacionar ambas posturas. Por ejemplo, Diéguez Lucena (2005) considera que la segunda es una variante de la primera; para Gómez (1997), ambas tesis son constitutivas del determinismo tecnológico; mientras que Parente (2006) considera que ambas se pueden distinguir, siendo posible aceptarlas o rechazarlas de manera independiente.
} 
moderno es antagónico e irreconciliable con la libertad humana (Kaczynski, 2008). Lewis Mumford, otro filósofo crítico con la tecnología, también contempla en el pasaje de la tecnología tradicional a la moderna este cambio de rol en la humanidad. Mientras que las herramientas y armas utilizadas antiguamente eran medios para conquistar la naturaleza, con la tecnología moderna el hombre es separado completamente de esta, convirtiéndose en un "animal pasivo, que sirve a la máquina” (Mumford, 2014, 381). Acorde a una perspectiva esencialista, la relación que estas posiciones señalan entre tecnología moderna y esclavitud no es accidental, sino que el fenómeno es "necesario e inevitable en cualquier sociedad tecnológicamente avanzada” (Kaczynski, 2008, 55).

La autonomía, el determinismo tecnológico y sus efectos alienantes, al ser propiedades esenciales - y por lo tanto inherentes- de la tecnología, hacen que el esencialismo tecnológico considere la posibilidad de evitarlos prácticamente imposibles y la opresión tecnológica inexorable (Gómez, 1997). Así, las alternativas y esperanzas para superar los males de la tecnología son cifradas en un evento sobrenatural, como expresa el propio Ellul (1964) o —de manera más célebre- Martin Heidegger en su sentencia "solo un dios puede salvarnos" (Feenberg, 2013); o bien en un evento catastrófico, como una guerra o revolución que derribe todo el sistema (Kaczyinski, 2008).

\subsection{En el imaginario popular}

Lejos de quedar reducida a un círculo de intelectuales, esta manera de entender la tecnología es parte también del imaginario popular, lo que se hace patente en clásicos del cine de ciencia ficción, que retoman varios de los lineamientos planteados hace más de 150 años por Butler. Por ejemplo, en la saga de películas Matrix se plantea un mundo donde la humanidad ha sido sometida por las computadoras, que han superado en capacidad e inteligencia al ser humano. Este es reducido a una batería, una fuente de alimento para las máquinas, como le es señalado al protagonista. Lejos de ser conscientes de esta situación, las personas viven alienadas en un mundo de estímulos falsos que aseguran la continuidad de la estructura tecnológica. Dicho sistema solo pareciera poder ser modificado por la acción externa de una singularidad, una deidad, encarnada en la figura mesiánica del protagonista. Un escenario similar es planteado en la serie de películas Terminator, donde las máquinas declaran la guerra a la humanidad. Aquí, aparte de la también mesiánica resistencia a la tecnología, la solución encontrada — en la segunda entrega de la saga - para salvar a la humanidad resalta el planteo determinista tecnológico de la película: solo puede evitarse la destrucción de la raza humana si se destruye un brazo robótico y un chip, primeros elementos de una serie de innovaciones, que llevarían indefectiblemente a la creación de las máquinas y su rebelión. 


\subsection{Principales rasgos del esencialismo tecnológico}

Un último aspecto que nos interesa resaltar de las perspectivas esencialistas en torno a la tecnología es el hecho de que la misma suele ser considerada como un ente monolítico y un fenómeno totalmente cerrado e inmutable (Franssen et al., 2018). El núcleo de dicha entidad, además, sería malvado o monstruoso (Niiniuluoto, 1990; Pellegrini, 2013). Para estas perspectivas, como señala Feenberg (2013), la modernidad está caracterizada por una única forma de acción técnica, que amenazaría todo valor no técnico. En oposición a la neutralidad del optimismo e instrumentalismo tecnológico, es adoptada una perspectiva sustancialista, donde la tecnología posee un sesgo valorativo que permearía y estructuraría toda la sociedad (Feenberg, 2013). Además, se enfrenta a dicha entidad monolítica con un segundo tipo de tecnología alternativa, que estaría libre de los vicios de la primera.

Heidegger, por ejemplo, en su ensayo La pregunta por la técnica (Heidegger, 1958), se propone buscar la esencia de la tecnología moderna, y para hacerlo la distingue y enfrenta a la tecnología tradicional, previa a la revolución industrial. La tecnología tradicional entablaría con la naturaleza una relación armoniosa, permitiendo descubrir la "verdad" de sus materiales (Feenberg, 2013, 19), donde la técnica no es solo tal, sino también arte. La tecnología moderna, en cambio, asume a la naturaleza de una manera reduccionista e instrumental, entendiendo a esta y al hombre solo como reservas - potenciales o actuales- de energía, para ser explotada y acumulada (Mitcham, 1989). Esto es lo que diferencia a la tecnología moderna de la tradicional. Este modo de relacionarse con la naturaleza es contrastado por Heidegger con la poiesis del artesano, que en su manipulación de los materiales entabla una armonía con estos y la naturaleza, que le permite revelar su esencia (Feenberg, 2013). La naturaleza, además, se convierte no en simple reserva, sino en objeto de respeto y asombro, algo que escapa al reduccionismo propio del desocultamiento que permite la tecnología. En contraste, la monstruosidad de la tecnología moderna corrompe esta perspectiva de la naturaleza, concibiéndola como un mero recurso. Esta relación, que Heidegger considera nociva, estará más allá de la voluntad del hombre, ya que tiene su origen en una actitud tecnológica, parte esencial de la tecnología (Mitcham, 1989). Debido a este carácter que escapa de la voluntad humana, ya que le es impuesto, pareciera imposible utilizar la tecnología para otra cosa que no sea entablar esta relación destructiva y reduccionista con la naturaleza. Para ilustrar esto, Heidegger compara un molino con una central eléctrica. El molino - propio de la tecnología tradicional-complementa y resalta las características del paisaje, así como obtiene su energía transfiriendo un movimiento que ya estaba en el viento o el agua. En cambio, la central violenta el paisaje, ya que no existe ningún tipo de armonía y su estructura es homogénea y se atiene a la lógica interna de la planta de energía. Al mismo tiempo, transforma a la materia de una manera mucho más radical y obtiene energía del interior mismo del carbón (Mitcham, 1989). Todo esto, 
considera Heidegger, violenta la naturaleza y oculta al ser. Todos, incluidos los humanos, pasamos a ser objetos y reservas disponibles de la técnica, cuya esencia es "la planificación metódica del futuro" (Feenberg, 2013, 18).

Además, para Heidegger tampoco importa de qué tecnología específica estemos hablando, todas en último término presentan el mismo problema insuperable, ya que todas se desarrollan en el mismo marco. De esta manera, señala una esencia en la tecnología, lo que le permite considerarla como una totalidad monolítica. Así, juzga y analiza la tecnología en una perspectiva tan general y abstracta que todas entablan -inherentemente y más allá de la voluntad humana - la misma relación reduccionista, violenta e instrumental con la naturaleza y el ser. No importa la tecnología particular de la que estemos hablando, ni el contexto social e histórico en que estas se desarrollen, todas tenderán a someter a la humanidad, convirtiéndola en objeto de control y simple reserva de energía. Las circunstancias y características particulares de cada tecnología son eclipsadas y consideradas meros accidentes frente a estos rasgos definitorios, a tal punto que para Heidegger no hay diferencia entre las técnicas agrícolas modernas y las utilizadas en el genocidio nazi (Rockmore, 1992). Como señala Feenberg, "el argumento de Heidegger está desarrollado en un nivel tan alto de abstracción que literalmente no puede discriminar entre electricidad y bombas atómicas, técnicas agrícolas y el holocausto" (Feenberg, 2013, 20).

Coincidiendo con Heidegger, Lewis Mumford distingue también dos tipos de tecnología: la politécnica (o biotécnica) y la monotécnica (también llamada autoritaria o megamáquina). La primera estaría orientada a la vida, en armonía con esta y un espíritu democrático; mientras que la segunda se orienta al poder, basada en la ciencia y dirigida a la expansión económica, material y militar (Mumford, 2014). También es patente - como en Heidegger- la romantización y la separación del hombre, a partir de la tecnología moderna, de la naturaleza y los aspectos estéticos y espirituales. La naturaleza ya no es algo apreciable sino como recurso. En cambio, la biotécnica permitiría encontrar una vida más llena de significado, que la tecnología moderna impide. Mumford identifica la esencia de la tecnología moderna en tiempos tan lejanos como hace 5000 años atrás, en el antiguo Egipto de los faraones, y considera que dicha esencia se mantuvo intacta. Esto ayuda a subrayar el carácter esencialista, en cuanto la megamáquina, no importa en qué lugar o tiempo se encuentre, siempre tendrá las mismas consecuencias nefastas. Al igual que Heidegger, este modo amplio de caracterizar a la monotécnica, permite a Mumford trazar correlaciones y equivalencias entre tecnologías en principio muy alejadas una de otra, como por ejemplo, las pirámides y los cohetes espaciales (Mumford, 2014).

Ellul, por su parte, distingue entre las acciones técnicas y el ya mencionado fenómeno técnico. Las primeras son múltiples, tradicionales y limitadas por los contextos en que se desarrollan. El fenómeno técnico, en cambio, se constituye en la única manera de fabricar y utilizar artefactos "que tienden a dominar e 
incorporar en sí mismo todas las otras formas de la actividad humana" (Mitcham, 1989, 79). Ellul prefiere a las acciones técnicas por su pluralidad y humanismo, en contra de la alienación que provoca el segundo. De manera similar, Kaczynski diferencia entre las tecnologías de pequeña escala y la tecnología dependiente de organizaciones, siendo esta última la modalidad dominante desde la revolución industrial (Kaczynski, 2008). A modo de ideal positivo, como contrapunto de esta y en armonía con las tecnologías de pequeña escala, Kaczynski propone el concepto de naturaleza salvaje. Tanto en Ellul como en Kaczynski, es explícito también el abordaje holista, que hace que ambos tomen a la tecnología moderna como un sistema unificado que debe ser rechazado como un todo (Ellul, 1990; Kaczynski, 2008).

En síntesis, el esencialismo tecnológico suele considerar a la tecnología como libre de todo condicionamiento social, ateniéndose a una lógica interna, inherente a sí misma. Dicha autonomía terminaría sofocando y eliminando la autonomía humana, vía el determinismo tecnológico. Además, la tecnología - no importa de qué caso concreto estemos hablando-siempre tendrá consecuencias funestas y perjudiciales, superando todo beneficio aparente que en principio pudiera traer. La presión tecnológica es inexorable, la maldad habitaría el interior de la tecnología, o bien esta funcionaría a modo de una caja de Pandora, como castigo a la humanidad por cruzar alguna barrera o límite de la naturaleza (Pellegrini, 2013, 41). En ambos casos, se identifican tanto posturas mistificantes (Katz, 1998) como una imagen de la tecnología monolítica, donde las circunstancias particulares y el diseño de la misma no son importantes ni afectan el núcleo duro y trágico de esta. Por último, dicho carácter esencial es enfatizado al enfrentar la tecnología moderna con algún tipo de alternativa benévola y libre de estos vicios. En estos planteos, la alternativa encarna todo lo contrario a la tecnología moderna y en armonía con la naturaleza, que pareciera ser considerada desde una perspectiva romántica, como reserva moral o de belleza intrínseca. Los puntos anteriores llevan a rechazar a la tecnología como un todo, sin posibilidad de discutir, criticar e intervenir en su diseño.

Desarrollados los principales puntos sobre la concepción esencialista de la tecnología, cerraremos el trabajo analizando un caso concreto - la controversia en torno a los cultivos transgénicos- y de qué manera la perspectiva esencialista influye en su planteo.

\section{El caso de los OGM}

Desde las primeras técnicas de manipulación del ADN, la ingeniería genética ha estado en el foco de diversas controversias que han traspasado las fronteras de la comunidad científica para desplegarse en el ámbito más amplio de la sociedad. Thompson (2014) se ha referido a la controversia en torno a la ingeniería genética como un "problema retorcido", ya que pone valores importantes en juego, los 
hechos están envueltos en incertezas, las alternativas y perspectivas son incompatibles entre sí y no existe ni siquiera un acuerdo fundamental sobre cuál es el problema. La producción y utilización de cultivos transgénicos es el centro de una discusión particularmente candente, desde su implementación en la actividad agropecuaria en la década de 1990 (Thompson, 2009; Pellegrini, 2013). Como explica Scott (2018), mientras que las empresas y principales actores interesados en el desarrollo de los cultivos transgénicos suelen adoptar un discurso optimista tecnológico y consideran a la tecnología como meramente instrumental, desde los sectores que se oponen se despliega un discurso pesimista tecnológico. En este apartado nos interesa centrarnos en este último enfoque y analizar el modo en que el esencialismo tecnológico, descrito en la sección anterior, subyace a la representación que se construye sobre los cultivos transgénicos en particular, y a los organismos genéticamente modificados (OGM) en general ${ }^{4}$.

Douglas Allchin señala que el esencialismo biológico está cifrado en muchas críticas y temores respecto a los OGM, al considerar que el cambio en los genes de un organismo alteraría su identidad como especie, considerando a las especies como entidades fijas y con un propósito definido (Allchin, 2014). Por esta línea circulan algunos de los argumentos de la ONG Greenpeace, en cuyo sitio web oficial, en la sección titulada ¿Por qué se opone Greenpeace a la liberación de Organismos Modificados Genéticamente (OMG) al medio ambiente?, se explica que "la diferencia fundamental con las técnicas tradicionales de mejora genética es que [la ingeniería genética moderna] permite franquear las barreras entre especies para crear seres vivos nuevos que no existían en la naturaleza" (Greenpeace, s.f.). Así, en este tipo de críticas, la modificación de genes amenazaría la identidad de una especie y generaría algo ajeno a lo "natural" (Allchin, 2014; Blancke et al., 2015). Acorde a esto, las encuestas sobre percepción de transgénicos realizadas por el Parlamento Europeo, muestran que cuanto mayor es la distancia entre las especies, menos aceptada es la modificación por el público general (Ankeny y Bray, 2012).

Sin embargo, nos interesa centrarnos en otro aspecto de estas críticas, donde también es posible identificar una perspectiva esencialista, pero esta vez en torno a la concepción de la tecnología, aspecto menos explorado en estas posturas. Según esta perspectiva, los OGM son conceptualizados como entidades autónomas y una fuente de riesgo y peligro inherente, independientemente de las características particulares del organismo, sus modificaciones o el contexto en el cual se presente. En este sentido, Greenpeace señala que "la contaminación genética es una de las grandes amenazas para el medio ambiente, debido a que una vez liberados los OGM no pueden ni ser controlados ni retirados" (Greenpeace, s.f.).

\footnotetext{
${ }^{4}$ Organismo transgénico y OGM no son términos equivalentes. El primero hace referencia a los organismos a los que se han insertado uno o más genes de otra especie, mientras que OGM es un término más amplio, que hace referencia a todo organismo que contiene una manipulación genética.
} 
La contaminación genética es uno de los principales argumentos en contra de los OGM, y se refiere a los riesgos que implica la introducción de transgenes en cultivos que originalmente no son transgénicos. Mediante entrecruzamientos espontáneos ${ }^{5}$, las construcciones genéticas de un cultivo transgénico se "escaparían" a otras especies "naturales" modificándolas inevitablemente. En un argumento similar, no ya los transgenes, sino las variedades transgénicas como un todo son consideradas especies invasivas (Paull, 2018). En ambos casos, se busca resaltar que, ya sean los genes o los OGM, por su propia naturaleza, tenderían a invadir los ecosistemas, reproduciéndose de manera descontrolada, más allá del manejo e intención humana. Mientras que la idea de que la tecnología cobra vida propia y gana la capacidad de reproducirse por sí misma es una metáfora recurrente en el esencialismo tecnológico para señalar su carácter autónomo, en la crítica a los OGM el carácter metafórico se pierde para interpretarse en un sentido literal ${ }^{6}$. El cuadro que describen estas miradas es el de una naturaleza estática que viene a ser alterada por un cultivo transgénico, que la perjudicaría inevitablemente porque la contaminación estaría dentro de su esencia y no se podría controlar.

Otro argumento en contra de los cultivos transgénicos que recurre a la idea de autonomía tecnológica es ubicar a estos como un eslabón más en una cadena de innovaciones que tienen como único objetivo resolver problemas que las otras tecnologías anteriores han creado. Según esta postura, la revolución biotecnológica que permitió la creación de variedades transgénicas es la respuesta a los problemas que generó la Revolución Verde entre las décadas de los 60' y 80' (Bravo, 2020). La tecnología agrícola no se orientaría a resolver problemas reales, sino que "los crea con el fin de desarrollar nuevos artefactos tecno-científicos destinados a solucionar los problemas creados por los primeros, y que a su vez generan nuevos" (Bravo, 2020,15). De esta manera, la ingeniería genética sería producto de una lógica y necesidad inherente al propio desarrollo tecnológico, en términos similares a los de Ellul, para quien la tecnología engendra más tecnología (Dusek, 2006).

Otra posición esencialista en torno a los cultivos transgénicos es la identificación de estos como fuente de todos los problemas sociales y ambientales relacionados al ámbito agrícola. Por ejemplo, el Grupo de Reflexión Rural ${ }^{7}$ expresa que "la biotecnología, el agronegocio, los transgénicos, los agrotóxicos, destruyen la biodiversidad, envenenan los ecosistemas e impactan directamente sobre nuestras poblaciones" (citado en Pellegrini, 2013, 40). Los transgénicos son asumidos

\footnotetext{
${ }^{5}$ Por ejemplo, polinización cruzada, mezcla de semillas, esparcimiento de semillas, etc.

${ }^{6}$ Diéguez Lucena, en referencia a la incapacidad para controlar la técnica que señala la autonomía tecnológica, expresa que "por el momento, no se refiere al descontrol de los aparatos y artefactos" (Diéguez Lucena, 2005, 79). Sin embargo, en el caso de los OGM, los argumentos en torno al escape y contaminación genética, sí parecieran ir en este sentido.

${ }^{7}$ Organización argentina que tiene como uno de sus principales objetivos la crítica a lo que llama "el modelo agrario biotecnológico".
} 
así como un agente de destrucción en sí mismos, sin importar el perfil particular de cultivo transgénico del que se trate ni su forma de regulación. En el mismo tenor, Soledad Barutti (2013) hace un repaso de la utilización de la soja transgénica resistente a glifosato en Argentina y sus consecuencias, donde la misma pareciera tener una agencia directa sobre estos fenómenos. Expresa, por ejemplo, que "la soja arrasó con el 14 por ciento de los bosques nativos que quedaban en pie" (Barruti, 2013, 75). Más allá del carácter autónomo que se les otorga a los cultivos transgénicos, este tipo de críticas también poseen un sesgo determinista tecnológico, ya que los OGM parecieran ser los que estructuran y condicionan todas las demás esferas del ámbito económico, social y ambiental.

En un libro en el que se proponen el abordaje de diversas controversias sociocientíficas, las biólogas y educadoras Alicia Massarini y Adriana Schnek —ambas abiertamente en contra de los OGM-dedican uno de sus capítulos a los cultivos transgénicos (Massarini y Schnek, 2015). En el mismo, puede verse de una manera bastante gráfica cómo una gran diversidad de problemas irradiaría directamente de los OGM (Imagen 1).

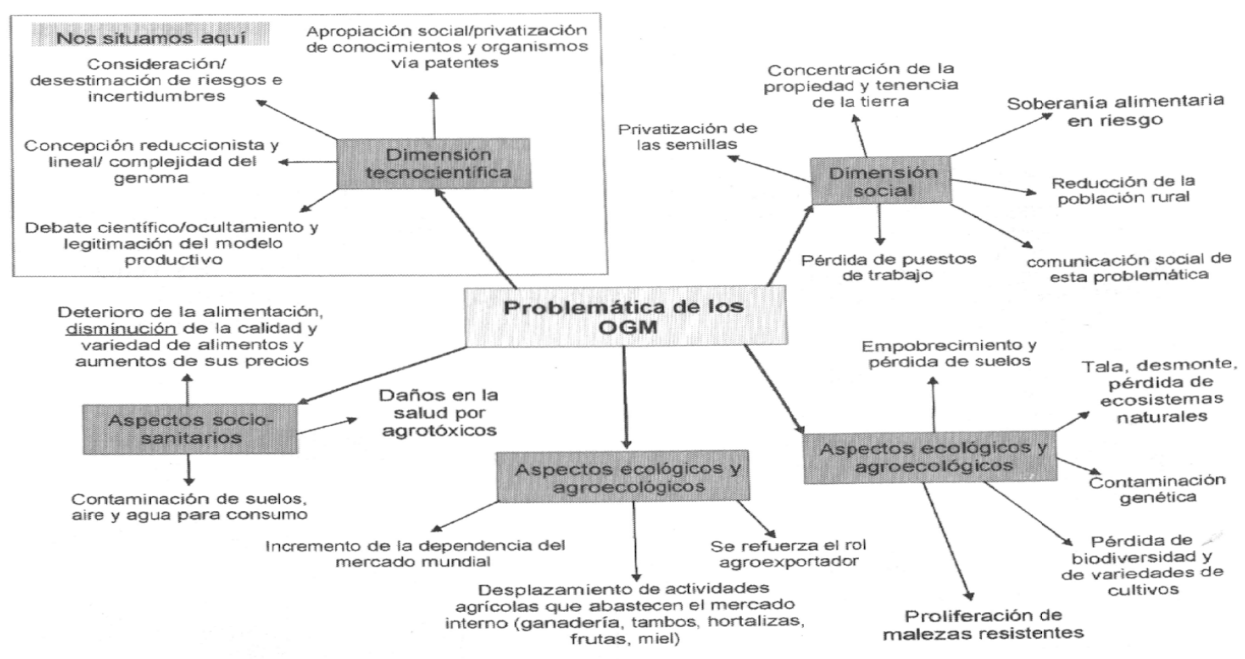

Imagen 1. Mapa conceptual de la controversia sobre OGM, en propuesta didáctica de Ciencia entre todxs (Massarini y Schnek, 2015, 139).

De esta manera, los OGM se presentan como origen de los problemas sociales, ya que el gráfico pareciera sostener que los OGM generarían la pérdida de puestos de trabajo y la concentración de la propiedad y tenencia de la tierra; pondrían en riesgo la soberanía alimentaria; reducirían la población rural; empobrecerían los suelos; generarían talas, desmontes y pérdida de ecosistemas naturales, biodiversidad y variedad de cultivos; provocarían la contaminación genética, la proliferación de malezas resistentes y dańos a la salud por agrotóxicos, llevando a la contaminación de suelos, aire y agua; e incrementarían la dependencia del 
mercado mundial, reforzando el rol agroexportador y desplazando otras actividades agrícolas. En el mismo texto, reforzando la postura esencialista, se expresa que "los cultivos genéticamente modificados traen aparejados impactos (...) que no pueden ser entendidos solo como resultado de un manejo inadecuado, sino que atañen a la propia naturaleza y concepción de la tecnología diseñada" (Massarini y Schnek, 2015, 150).

Acorde a la concepción monolítica de la tecnología, las autoras condenan la tecnología que los produce como un todo, ya que consideran que "la transgénesis (...) es una técnica agresiva y peligrosa para el ambiente, la alimentación y la salud" (Clase de ciencia, 2015). Massarini, en la misma línea, expresa que "el problema con esta tecnología es que no puede ser usada para el bien porque está diseñada, pensada y usada para maximizar la ganancia económica. Todo lo demás son daños colaterales" (Viola, 2015). Desde este enfoque, debe evaluarse a la ingeniería genética como un todo, sin poder distinguir sus diversos usos y aplicaciones concretas, ni contextualizarla en sus desarrollos particulares, pues es pensada como la encarnación del Mal.

Más allá de los ejemplos concretos aquí dados, esta estrategia y enfoque a la controversia es ampliamente utilizada por diversos movimientos ambientalistas y opositores a los OGM (Pellegrini, 2007; Allchin, 2014; Harker, 2015). Muchos de quienes mantienen esta perspectiva monolítica en torno a los OGM, los enfrentan a un modelo agrícola alternativo, llamado "agricultura ecológica". Mientras que la llamada "agricultura transgénica" es considerada una tecnología ya cerrada y con una lógica industrial que presenta una misma solución universal para cualquier situación, la agricultura ecológica es abierta y flexible, gracias a los conocimientos locales, de carácter específico (Gunzmán, 2006). A su vez, estos conocimientos poseerían sus raíces en saberes tradicionales y de carácter eminentemente empírico, en contraste a la lógica científica y tecnológica moderna que impregnaría a los transgénicos, desarrollados en base a una abstracción y teorización insensibles a las especificidades culturales y ecológicas de cada lugar (Guzmán, 2006). Estas características diametralmente opuestas, llevarían a que la agricultura ecológica solucionara todos aquellos males cuyo origen reconocen en los OGM, y así lograr tanto la inocuidad y calidad de los alimentos, como la igualdad y seguridad económica de los campesinos; tomar en consideración los desafíos y problemas ambientales; detener el éxodo rural y mejorar las condiciones de vida y trabajo, así como la igualdad de oportunidades (López, 2007; Pellegrini, 2009). Desde esta perspectiva, los transgénicos parecieran ser de alguna manera esencialmente distintos a otras formas de intervención agrícola, a tal punto que muchos defensores de la agricultura ecológica, consideran que los OGM son incompatibles con dicho modelo, llevando a caminos de desarrollo y producción diametralmente opuestos (Amigos de la tierra et al., 2014; Ecologistas en acción, 2014; Bioeco actual, 2018). 
La mirada esencialista en torno a los OGM lleva a estos al centro de la escena y los propone como el problema principal, a partir del cual se derivan todos los demás. Esa lógica lleva a pensar, implícita o explícitamente, que una vez eliminados los OGM, el camino estaría allanado para una agricultura sustentable y fuente de igualdad y desarrollo social, como la politécnica de Mumford o las tecnologías de pequeńa escala de Kaczynski. De manera paralela, lleva a la imposibilidad de pensar ese escenario en presencia de los OGM, sean cuales sean estos. De esta forma, toda la discusión y sus aristas quedan reducidas a la cuestión de si aceptar o rechazar los cultivos transgénicos, simplificando la discusión acerca de los diversos problemas socioambientales planteados. $\mathrm{Al}$ respecto, Harker expresa que "quizás la característica más desafortunada de las conversaciones que rodean a los OGM es la implicación que o bien todos comparten los mismos riesgos profundamente preocupantes o bien todos son seguros" (Harker, 2015, 426).

\section{Conclusiones}

En el presente trabajo hemos buscado describir los principales rasgos del estilo de pensamiento esencialista vinculado a la tecnología. Comenzamos por mostrar las raíces filosóficas del esencialismo aristotélico. A partir de este, mostramos que sus principales tesis subyacen al planteo de muchas posturas pesimistas de la tecnología, provenientes tanto de la cultura popular como de la filosofía de la tecnología. Según esta perspectiva, la tecnología moderna se desarrollaría de manera autónoma en base a una lógica propia y determinaría la estructura y organización de las demás esferas de la sociedad. Además, para el esencialismo la tecnología es entendida como un todo sistémico que tiene como característica esencial la alienación del ser humano, así como la destrucción de la naturaleza. En oposición a esta entidad monolítica, se encontraría una alternativa benévola, en armonía con el desarrollo y la realización humana, así como con la naturaleza.

Lejos de ser una caracterización inocua, este modo de entender la tecnología tiene consecuencias concretas en la forma de plantear y abordar las controversias públicas en torno a las innovaciones tecnológicas. En este sentido, desarrollamos, a modo de ejemplo, el caso de los cultivos transgénicos, mostrando cómo en su rechazo frecuentemente subyace una perspectiva esencialista. Dicha perspectiva, así como los enfoques esencialista-optimistas, empobrecen la discusión y reducen drásticamente la complejidad de la problemática.

Creemos que una mirada más contextualizada, que considere el carácter contingente de la tecnología y el contexto en el cual se desarrolla, permite análisis más matizados y menos maniqueos en torno a los desarrollos tecnológicos, que escapan así a las perspectivas fatalistas y apocalípticas. En este sentido, los estudios sociales de la ciencia y tecnología (Thomas y Buch, 2008), la teoría crítica de la tecnología (Feenberg, 2013; Tula Molina y Giulano, 2015) y el giro empírico en la filosofía de la tecnología (Franssen et al., 2016) habilitan un abordaje de la 
tecnología y su relación con la sociedad más complejo, permitiendo considerar la diversidad de desarrollos, interpretaciones y posibilidades que encierra no ya la tecnología, entendida como un todo monolítico, sino las diversas tecnologías concretas ubicadas y las particularidades de cada una de estas en función de sus condiciones específicas de desarrollo. Finalmente, la caracterización del estilo de pensamiento esencialista puede ayudar a comprender los aspectos culturales que condicionan las formas de pensar las tecnologías actuales.

\section{Agradecimientos}

A la Agencia Nacional de Promoción de la Investigación, el Desarrollo Tecnológico y la Innovación (Ministerio de Ciencia, Tecnología e Innovación, Argentina) por el financiamiento PICT-2018-1997 para el estudio de controversias científicas.

\section{Referencias bibliográficas}

Achterhuis, Hans (2001). American philosophy of technology: The empirical turn. Indiana University Press.

Ainsworth, Thomas (2020). Form vs. Matter. The Stanford Encyclopedia of Philosophy, Edward N. Zalta (ed.). https://plato.stanford.edu/archives/sum2020/entries/ form-matter/

Allchin, Douglas (2014). Organisms, modified, genetically. The american biology Teacher, 76(9), 639-641.

Amigos de la tierra, COAG, Ecologistas en Acción, Greenpeace y CECU (2009). Implicaciones socio-económicas de la introducción de OMGs en el mercado para su cultivo. https://www.ecologistasenaccion.org/wp-content/uploads/adjuntosspip/pdf_Informe_implicaciones_socioeconomicas_transgenicos.pdf

Ankeny, R. A., Bray, H. (2018). Genetically modified food. En Anne Barnhill, Mark Budolfson and Tyler Doggett (Eds.), The Oxford Handbook of Food Ethics (pp. 95-111). Oxford: Oxford University Press.

Barnes, Barry, Bloor, David (1982). Relativism, Rationalism and the Sociology of Knowledge. En M. Hollis \& S. Lukes (Eds.), Rationality and Relativism (pp. 2147). Cambridge: MIT Press.

Barutti, Soledad (2013). Un problema en carne viva. Mal comidos. Buenos Aires: Planeta.

Bioeco Actual (2018). En Agroecología no caben los transgénicos. 4 de Abril. https:// www.bioecoactual.com/2018/04/04/agroecologia-no-caben-los-transgenicos/

Blancke, S., Van Breusegem, F., De Jaeger, G., Braeckman, J., Van Montagu, M. (2015). Fatal attraction: the intuitive appeal of GMO opposition. Trends in plant science, 20(7), 414-418. 
Bloor, David (2011). Relativism and the Sociology of Scientific Knowledge. En S. Hales (Ed.), A Companion to Relativism (pp. 433-455). Oxford: Wiley-Blackwell.

Bravo, Elizabeth (2020). La instrumentalización de las leyes naturales para la acumulación del capital. El caso de la biología molecular. Ciencia Digna, 1(1), 8-17.

Butler, Samuel (1914). Darwin Among The Machines. En R. A. Streatfeild (Ed.), A first year in Canterbury Settlement with other early essays (pp. 179-185). Londres. J. Cape.

Butler, Samuel (2012). Erewhon o Al otro lado de las montañas. Madrid: Akal.

Capanna, Pablo (1966). El sentido de la ciencia ficción (No. 1). Buenos Aires: Editorial Columbia.

Clase de ciencia (2015). La Vaca. 23 de Septiembre. http://www.lavaca.org/mu92/clasede-ciencia/

Cohen, S. Marc (2020). Aristotle's Metaphysics. The Stanford Encyclopedia of Philosophy, Edward N. Zalta (ed.). https://plato.stanford.edu/archives/fall2020/entries/ aristotle-metaphysics/

Collins, H. (1981). What is TRASP? The Radical Programme as a Methodological Imperative. Philosophy of the Social Sciences, 11(2), 215-224.

Diéguez Lucena, Antonio (2005). El determinismo tecnológico: indicaciones para su interpretación. Argumentos de Razón Técnica, 8, 67-87.

Douglas, Mary (1998). Estilos de pensar. Barcelona: Gedisa.

Dusek, Val (2006). Philosophy of technology: An introduction (Vol. 90). Oxford: Blackwell.

Ecologistas en acción (2014). Los cultivos transgénicos ponen en peligro la agricultura ecológica. https://www.ecologistasenaccion.org/27916/los-cultivos-transgenicosponen-en-peligro-la-agricultura-ecologica/

Ellis, Brian (2002). Philosophy of nature: A guide to the new essentialism. Montreal, Quebec: McGill-Queen's Press-MQUP.

Ellul, Jacques (1964). The Technological Society. New York: Alfred A. Knopf.

Ellul, Jacques (1981). A temps et a contretemps, (entrevista con Madeleine GanigouLagrange). Le Centurión, p. 155.

Ellul, Jacques (1990). The technological bluff. Grand Rapids, MI: Eerdmans.

Ellul, Jacques (2014). The autonomy of the Technological Phenomenom. En R.C. Scharff y Val Dusek (Eds.), Philosophy of Technology (pp. 430-441). UK: John Wiley \& Sons.

Feenberg, Andrew (2013). Del esencialismo al constructivismo: la filosofía de la tecnología en la encrucijada. Hipertextos, 1(1), 15-57. 
Franssen, M., Lokhorst G. J., van de Poel I. (2018). Philosophy of Technology. The Stanford Encyclopedia of Philosophy, Edward N. Zalta (Ed.). https://plato.stanford. edu/archives/fall2018/entries/technology/

Franssen, M., Vermaas, P. E., Kroes, P., Meijers, A. W. (Eds.). (2016). Philosophy of technology after the empirical turn. Cham: Springer International Publishing.

Gómez, Ricardo (1997). Progreso, determinismo y pesimismo tecnológico. Redes, 4(10), 59-94.

Gómez Di Vincenzo, José Antonio (2012). Artilugios técnicos y máquinas de pensar: la reflexión acerca de la tecnología. En H. Palma y R. Pardo (Eds.), Epistemología de las Ciencias Sociales. Perspectivas y problemas de las representaciones científicas de lo social (pp. 129-151). Buenos Aires: Biblos.

Gómez-Lobo, Alfonso (1996). Exposición breve de la metafísica aristotélica. Estudios públicos, 62, 309-327.

Greenpeace (s.f.). ¿Por qué se opone Greenpeace a la liberación de Organismos Modificados Genéticamente (OMG) al medio ambiente? https://es.greenpeace. org/es/preguntas-frecuentes/por-que-se-opone-greenpeace-a-la-liberacion-deorganismos-modificados-geneticamente-omg-al-medio-ambiente/

Guzmán, Eduardo Sevilla (2006). Agroecología y agricultura ecológica: hacia una "re" construcción de la soberanía alimentaria. Agroecología, 1, 7-18.

Harker, David (2015). Creating scientific controversies: uncertainty and bias in science and society. Cambridge University Press.

Heidegger, Martin (1958). La pregunta por la técnica. Revista de filosofía, 5(1), 55-79.

Kaczynski, Theodore (2008). The Road to Revolution. Suiza: Xenia.

Kapp, Ernst (1998). Líneas fundamentales de una filosofía de la técnica. Teorema: Revista internacional de filosofía, 17(3), 111-118.

Kintz, Theresa (2014). The anarchist library. https://theanarchistlibrary.org/library/ theresa-kintz-interview-with-ted-kaczynski.lt.pdf

Klima, Gyula (2017). The Medieval Problem of Universals. The Stanford Encyclopedia of Philosophy, Edward N. Zalta (ed.). https://plato.stanford.edu/archives/win2017/ entries/universals-medieval/

Kuukkanen, Jouni-Matti (2016). Historicism and the failure of HPS. Studies in History and Philosophy of Science Part A, 55, 3-11.

Latour, Bruno (2000). On the partial existence of existing and non-existing objects. En L. Daston (Ed.), Biographies of scientific objects (pp. 247-269). Chicago: University of Chicago Press.

López, Xosé Armesto (2007). The concept of ecological agriculture and its suitability to foment rural sustainable development. Boletín de la AGEN, 43, 387-389. 
Massarini, Alicia, y Schnek, Adriana (2015). Ciencia entre todxs. Tecnociencia en contexto social. Una propuesta de enseñanza. 1 ed. Ciudad Autónoma de Buenos Aires: Paidós.

Mitcham, Carl (1989). ¿Qué es la filosofía de la tecnologia? Barcelona: Anthropos.

Mumford, Lewis (2014). Tool Users vs. Homo Sapiens and the Megamachine. En R.C. Scharff y Val Dusek (Eds.), Philosophy of Technology (pp. 381-388). UK: John Wiley \& Sons.

Niiniluoto, Ilkka (1990). Should technological imperatives be obeyed?. International Studies in the Philosophy of Science, 4(2), 181-189.

Oderberg, David S. (2007). Real essentialism. London: Routledge.

Parente, Diego (2006). Algunas precisiones sobre el determinismo tecnológico y la tecnología autónoma. Una lectura sobre la filosofía de Langdon Winner. Redes, 12(23), 79-102.

Paull, John (2018). Genetically modified organisms (GMOs) as invasive species. Journal of Environment Protection and Sustainable Development, 4(3), 31-37.

Pellegrini, Pablo Ariel (2007). Riesgo y contexto. Un análisis sobre el discurso del riesgo tecnológico en los movimientos sociales. Ciencia, docencia y tecnología, 18(35), 51-88.

Pellegrini, Pablo Ariel (2009). Knowledge, identity and ideology in stances on GMOs: The case of the Movimento Sem Terra in Brazil. Science \& Technology Studies, 22(1), 44-63.

Pellegrini, Pablo Ariel (2013). Transgénicos: ciencia, agricultura y controversias en la Argentina. Buenos Aires: Universidad Nacional de Quilmes.

Pellegrini, Pablo Ariel (2019). La verdad fragmentada: conflictos y certezas en el conocimiento. Buenos Aires: Argonauta.

Quintanilla, Miguel Á. (2017). Tecnologías entrañables: un modelo alternativo de desarrollo tecnológico. En M. A. Quintanilla, M. Parselis, D. Sandrone y D. Lawler (Eds.), Tecnologías entrañables (pp. 15-53). Madrid: Catarata.

Rockmore, Tom (1992). On Heidegger's Nazism and Philosophy. Berkeley: University of California Press.

Rodriguez-Pereyra, Gonzalo (2019). Nominalism in Metaphysics. The Stanford Encyclopedia of Philosophy, Edward N. Zalta (Ed.). https://plato.stanford.edu/ archives/sum2019/entries/nominalism-metaphysics/

Ross, William David (1981). Aristóteles. Trad. Diego F. Pró. Biblioteca de Filosofía. 2a ed. Buenos Aires: Charcas.

Scott, Dane (2018). Food, Genetic Engineering and Philosophy of Technology: Magic Bullets, Technological Fixes and Responsibility to the Future (Vol. 28). Cham: Springer. 
Shapin, Steven (1995). Here and Everywhere: Sociology of Scientific Knowledge. Annual Review of Sociology, 21, 289-321.

Thomas, Hernán, Buch, Alfonso (2008). Actos, actores y artefactos: sociología de la tecnologia. Bernal: Universidad Nacional de Quilmes.

Thomasson, Amie (2003). Realism and Human Kinds. Philosophy and Phenomenological Research, 67(3), 580-609.

Thomasson, Amie (2007). Artifacts and Human Concepts. En E. Margolis and S. Laurence (Eds.), Creations of the Mind: Essays on Artifacts and Their Representation (pp. 52-73). Oxford: Oxford University Press.

Thompson, P (2009). Philosophy of agricultural technology. En A. Meijers (Ed.), Philosophy of technology and engineering sciences (pp. 1257-1273). Amsterdam: North-Holland.

Thompson, Paul B. (2014). The GMO quandary and what it means for social philosophy. Social Philosophy Today, 30, 7-27.

Tula Molina, F., Giuliano, H. G. (2015). La teoría crítica de la tecnología: revisión de conceptos. REDES, 21(40), 179-214.

Viola, Natalia (2015). Transgénicos: la polémica está servida en tu mesa. La Gaceta. https://www.lagaceta.com.ar/nota/640141/sociedad/transgenicos-polemica-estaservida-tu-mesa.html

Winner, Langdon (1977). Autonomous technology: techniques-out-of-control as a theme in political thought. Cambridge: Massachusetts institute of technology. 